SUBJECT AREAS:

TOPOLOGICAL

INSULATORS

QUANTUM HALL

Received

17 October 2013

Accepted

5 November 2013

Published

6 December 2013

Correspondence and requests for materials should be addressed to

M.E. (ezawa@ap.t. u-tokyo.ac.jp)

\title{
High Spin-Chern Insulators with Magnetic Order
}

\author{
Motohiko Ezawa
}

Department of Applied Physics, University of Tokyo, Hongo 7-3-1, 1 13-8656, Japan.

As a topological insulator, the quantum Hall $(\mathrm{QH})$ effect is indexed by the Chern and spin-Chern numbers $\mathcal{C}$ and $\mathcal{C}_{\text {spin. }}$. We have only $\mathcal{C}_{\text {spin }}=0$ or $\pm \frac{1}{2}$ in conventional $\mathrm{QH}$ systems. We investigate $\mathrm{QH}$ effects in generic monolayer honeycomb systems. We search for spin-resolved characteristic patterns by exploring

Hofstadter's butterfly diagrams in the lattice theory and fan diagrams in the low-energy Dirac theory. It is shown that the spin-Chern number can takes an arbitrary high value for certain QH systems. This is a new type of topological insulators, which we may call high spin-Chern insulators. Samples may be provided by graphene on the $\mathrm{SiC}$ substrate with ferromagnetic order, transition-metal dichalcogenides with ferromagnetic order, transition-metal oxide with antiferromagnetic order and silicene with ferromagnetic order. Actually high spin-Chern insulators are ubiquitous in any systems with magnetic order. Nevertheless, the honeycomb system would provide us with unique materials for practical materialization.

. he quantum Hall $(\mathrm{QH})$ effect is one of the most fascinating phenomena in physics ${ }^{1-3}$. It is characterized by the topological index ${ }^{4}$. The integer QH state at the filling factor $v=n$ has the Chern number $n$. The concept of topological insulator stems from QH systems $s^{5,6}$. The topological insulator is characterized by the Chern number $\mathcal{C}$ and the $\mathbb{Z}_{2}$ index in the presence of the time-reversal symmetry, while by the Chern number $\mathcal{C}$ and the spin-Chern number $\mathcal{C}_{\text {spin }}$ when the spin $s_{z}$ is a good quantum number ${ }^{7-9}$. We investigate the spin-Chern number in $\mathrm{QH}$ systems. In the conventional monolayer QH system it takes $1 / 2$ and 0 alternately as up-spin and down-spin electrons fill Landau levels successively. The phenomenon occurs because the cyclotron energy $\hbar \omega_{\mathrm{c}}$ is always larger than the Zeeman energy $\Delta_{\mathrm{Z}}$ in general. Indeed, we have $\hbar \omega_{\mathrm{c}} \simeq 20.0 B \mathrm{~K}$ and $\Delta_{\mathrm{Z}} \simeq 0.296 B \mathrm{~K}$ in $\mathrm{GaAa}$ samples, where $B$ is the magnetic field in Tesla. In the bilayer QH system it can be at most 1 due to the layer degree of freedom ${ }^{3}$. There are no conventional QH states with higher spin-Chern numbers.

In this work we investigate an intriguing possibility to materialize QH states carrying higher spin-Chern numbers. For this purpose we make use of a state which contains either up-spin or down-spin electrons near the Fermi level in the absence of external magnetic field. Such a state is available in a system when it has strong exchange interactions produced by magnetic order. The magnetic order may be intrinsically present or introduced externally in the system. When the energy spectrum is split into Landau levels in magnetic field they are expected to carry high spinChern numbers, yielding a new type of topological insulators. We call them high spin-Chern insulators.

As realistic systems we analyze Dirac electrons in monolayer honeycomb systems. Dirac electrons are massless in graphene while they are massive in other materials. We have previously presented a generic Hamiltonian for honeycomb systems ${ }^{10}$, which contains eight interaction terms mutually commutative in the Dirac limit. Among them four contribute to the Dirac mass. The other four contribute to the shift of the energy spectrum. We are able to make a full control of the Dirac mass and the energy shift independently at each spin and valley by varying these parameters, and materialize various topological phases ${ }^{11,12}$. Without external magnetic field, the spin-valley dependent Chern number takes $\pm 1 / 2$. Thus we have only 16 types of topological insulators ${ }^{10}$. In this classification, the Chern number can only be $-2,-1,0,1,2$, while the spin-Chern number can be $-1,-1 / 2,0,1 / 2,1$. However, once we switch on magnetic field, the Chern number can take almost all integer values.

The magnetic order can be ferromagnetic or antiferromagnetic. Examples are given by graphene on the $\mathrm{SiC}$ substrate with ferromagnetic order, transition-metal dichalcogenides $\left(\mathrm{MX}_{2}\right)^{13-15}$ with ferromagnetic order, perovskite transitionmetal oxide grown on [111] direction (TMO) ${ }^{16,17}$ with antiferromagnetic order, and silicene ${ }^{18,19}$ with ferromagnetic order. It is interesting that the magnetic order is present intrinsically in $\mathrm{MX}_{2}$ and $\mathrm{TMO}$. In the absence of magnetic field, graphene, $\mathrm{MX}_{2}$ and $\mathrm{TMO}$ are trivial insulators while silicene is a quantum spin-Hall (QSH) insulator.

We explore Hofstadter's butterfly diagrams ${ }^{20-25}$ to see a global pattern of spin resolution. We also explore fan diagrams to see a detailed pattern of spin resolution in the low-magnetic field regime. We then calculate the Chern and spin-Chern numbers based on the bulk-edge correspondence ${ }^{21}$ and on the Kubo formula ${ }^{26,27}$. They show a 
(a) graphene

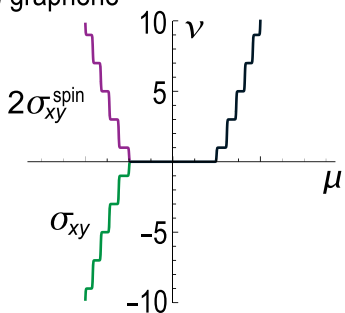

(c) TMO

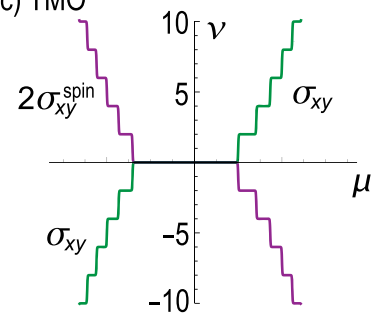

(b) $\mathrm{MX}_{2}$

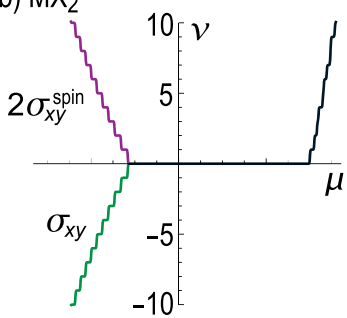

(d) silicene

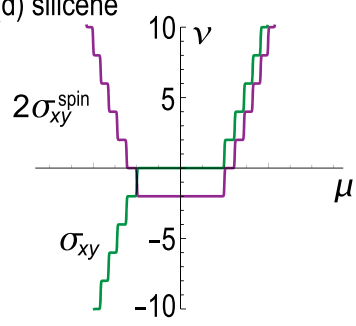

Figure $1 \mid$ Plateaux for the Hall conductivity and the spin-Hall conductivity for $|\boldsymbol{B}|<16$ Tesla. The vertical axis is the filling factor $v$, which is equal to $\sigma_{x y}$ (green) in unit of $e^{2} / 2 \pi \hbar$. We have also shown $2 \sigma_{x y}^{\text {spin }}$ (violet) in unit of $e / 2 \pi \hbar$. When they agree, the curves are in grey. The horizontal axis represents the chemical potential $\mu$. Graphene, dichalcogenide, TMO are trivial insulators at $v=0$, while silicene is a QSH insulator at $v=0$.

perfect agreement in this regime. Our main result is the prediction of high spin-Chern insulators that may have arbitrarily high spinChern numbers.

High spin-Chern insulators are actually ubiquitous. The condition is that the band structure has a finite region in the vicinity of the Fermi level where spins are perfectly polarized. Consequently, we would be able to generate them also in conventional QH systems, where electrons are described by the Schrödinger equation. A typical example is given by a square-lattice system, where we can show that high spin-Chern insulators arise precisely as in honeycomb systems. However, there exists no realistic monolayer square-lattice materials. We may also think of ordinary $\mathrm{QH}$ systems such as GaAs samples. It might be possible to introduce magnetic order into the system by doping $\mathrm{Mn}$ atoms since $\mathrm{Mn}$ atoms form a ferromagnetic order. However the mobility becomes drastically lower by the inhomogeneous doping of $\mathrm{Mn}$, and $\mathrm{QH}$ effects would hardly be observed. There exists a recent realization of ordinary $\mathrm{QH}$ effects on the interface of the oxides $\mathrm{MgZnO} / \mathrm{ZnO}^{28}$. Even in this material it is impossible to introduce the magnetic order since the electron layer is formed on the interface. On the contrary, there are realistic materials for monolayer honeycomb systems. They have very high mobility because samples have no impurities. They are excellent candidates for practical materialization of high spin-Chern insulators.

\section{Results}

The honeycomb lattice consists of two sublattices made of $A$ and $B$ sites. We consider a buckled system with the layer separation $2 \ell$

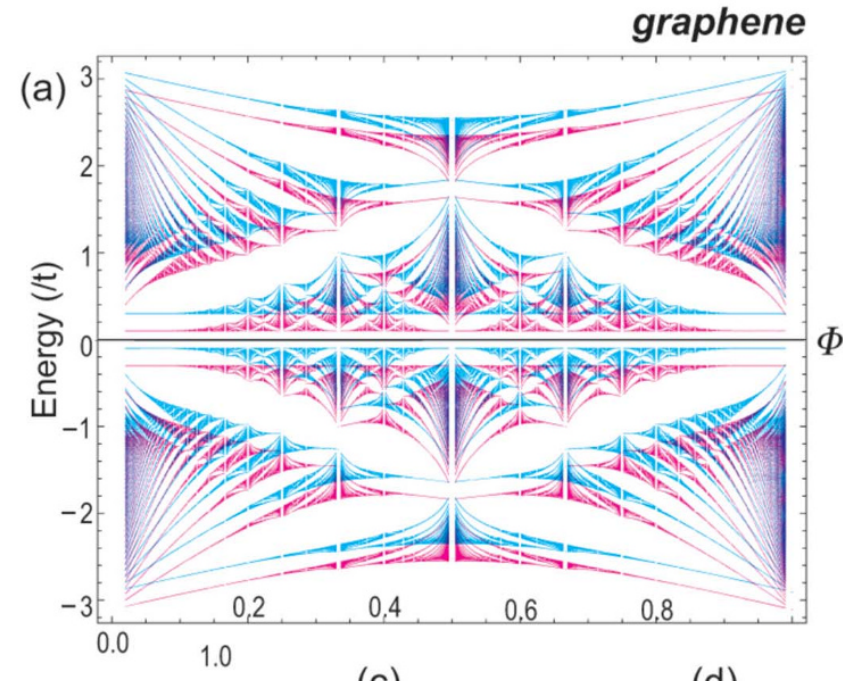

(b)

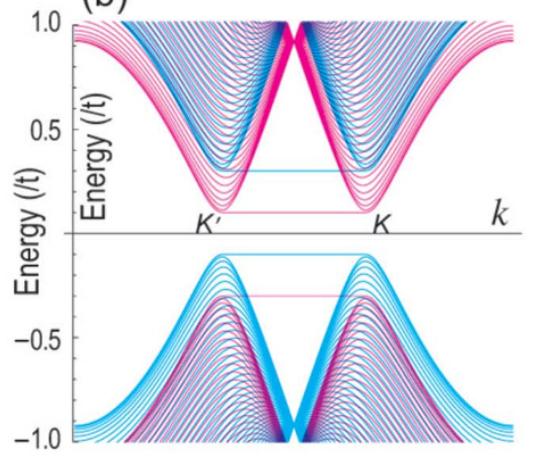

(c)

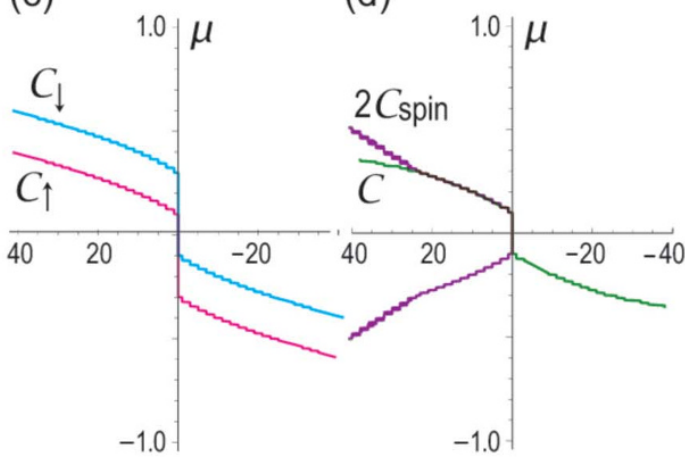

Figure $2 \mid$ Graphene with ferromagnetic order. We have set $\lambda_{V}=0.2 t$ and $\lambda_{\mathrm{X}}=0.1 t$ for illustration. The vertical axis is the energy in unit of $t$ in all figures. (a) Spin-resolved Hofstadter's diagram. The horizontal axis is the magnetic flux $\Phi$. We have taken $\Phi=p / q$ with $q \leq 100$. The contribution from up(down)-spin electrons is shown in magenta (cyan). (b) Band structure of a zigzag nanoribbon at $\Phi=0$. Spins are polarized near the Fermi level. The horizontal axis is the momentum $k$. (c) Topological number $\mathcal{C}_{s_{z}}$ calculated based on the Dirac formula (17) as a function of $\mu$ at $\Phi=1 / 1000$. It is found that $\left|\mathcal{C}_{s_{z}}\right|$ begins to increase monotonously when the bulk band having spin $s_{z}$ begins to be filled in (b). (d) Chern and spin-Chern numbers $\mathcal{C}$ (green) and $2 \mathcal{C}_{\text {spin }}$ (violet) as a function of $\mu$ derived based on (6) at $\Phi=1 / 1000$. We have taken $\mu$ as the vertical axis and Chern numbers as the horizontal axis. 


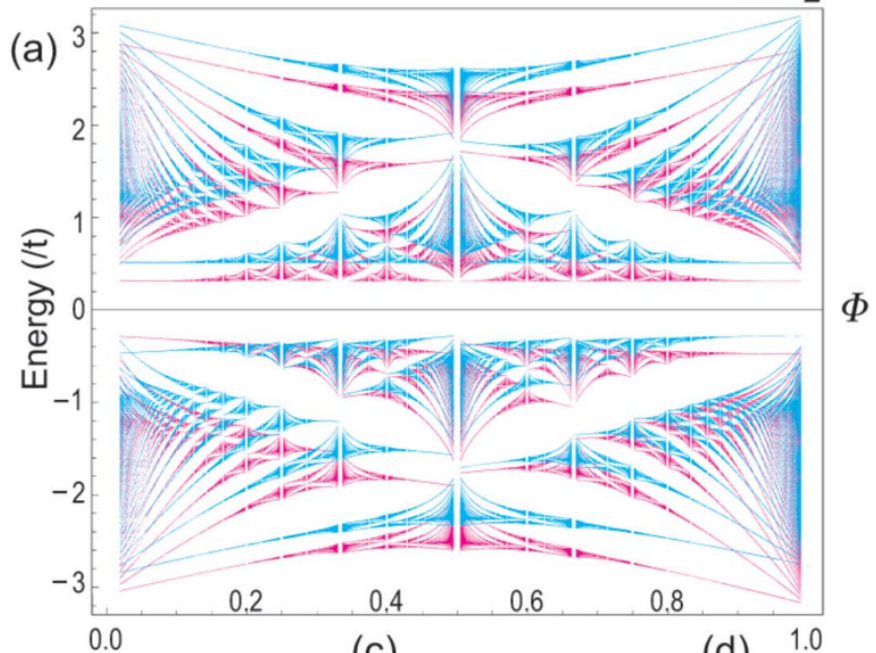

(b)

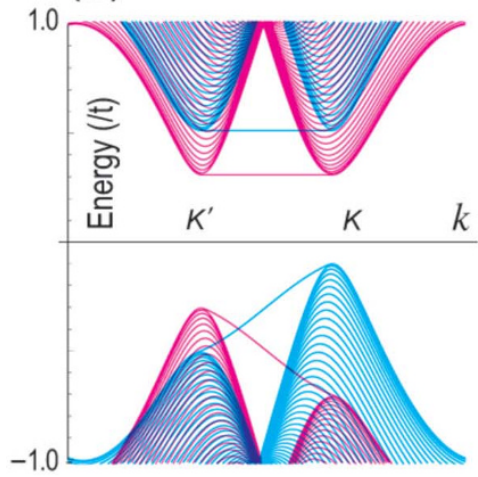

(c)

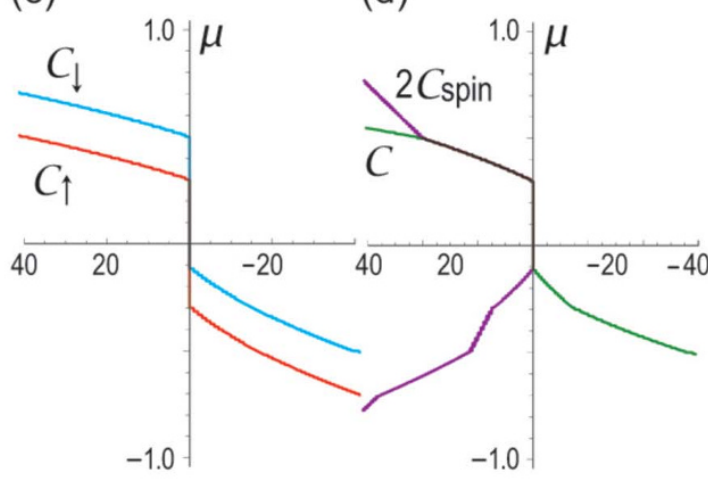

Figure $3 \mid$ Dichalcogenide. We have set $\lambda_{\text {SO }}=\lambda_{\text {SSO }}=0.1 t$ and $\lambda_{V}=0.4 t$ for illustration. See also the caption of Fig. 2.

between these two sublattices. The states near the Fermi energy are $\pi$ orbitals residing near the $K$ and $K^{\prime}$ points at opposite corners of the hexagonal Brillouin zone. The low-energy dynamics in the $K$ and $K^{\prime}$ valleys is described by the Dirac theory. In what follows we use notations $s_{z}=\uparrow \downarrow, t_{z}=A, B, \eta=K, K^{\prime}$ in indices while $s_{z}^{\alpha}= \pm 1$ for $\alpha=\uparrow \downarrow, t_{z}^{i}= \pm 1$ for $i=A, B$, and $\eta_{i}= \pm 1$ for $i=K, K^{\prime}$ in equations. We also use the Pauli matrices $\sigma_{a}$ and $\tau_{a}$ for the spin and the sublattice pseudospin, respectively.

We investigate the honeycomb system in perpendicular magnetic field $B$ by introducing the Peirls phase, $\Phi_{i j}=\frac{e}{h} \int_{r_{i}}^{r_{j}} A \cdot d r$, with $A$ the magnetic potential. Any hopping term from site $i$ to site $j$ picks up the phase factor $e^{2 \pi i \Phi_{i j}}$. The magnetic field is given by $B=2 \Phi / 3 \sqrt{3} a^{2}$ in unit of $e / h$, where $a$ is the lattice constant and $\Phi$ is the magnetic flux penetrating one hexagonal area. Note that $\Phi=1$ implies $B=1.6 \times$ $10^{5}$ Tesla in the case of graphene.

The relevant tight-binding model is given by ${ }^{10,29,30}$,

$$
\begin{aligned}
H & =-t \sum_{\langle i, j\rangle \alpha} e^{2 \pi i \Phi_{i j}} c_{i \alpha}^{\dagger} c_{j \alpha}-\mu \sum_{i \alpha} c_{i \alpha}^{\dagger} c_{i \alpha} \\
& +i \frac{\lambda_{\mathrm{SO}}}{3 \sqrt{3}} \sum_{\ll i, j \gg \alpha} s_{z}^{\alpha} e^{2 \pi i \Phi_{i j}} v_{i j} c_{i \alpha}^{\dagger} c_{j \alpha}+\lambda_{V} \sum_{i \alpha} t_{z}^{i} c_{i \alpha}^{\dagger} c_{i \alpha} \\
& +\lambda \mathrm{X} \sum_{i \alpha} s_{z}^{\alpha} c_{i \alpha}^{\dagger} c_{i \alpha}+\lambda_{\mathrm{SX}} \sum_{i \alpha} t_{z}^{i} s_{z}^{\alpha} c_{i \alpha}^{\dagger} c_{i \alpha}, \\
& +i \frac{\lambda_{\mathrm{SSO}}}{3 \sqrt{3}} \sum_{\ll i, j \gg \alpha} t_{z}^{i} s_{z}^{\alpha} e^{2 \pi i \Phi_{i j}} v_{i j} c_{i \alpha}^{\dagger} c_{j \alpha}
\end{aligned}
$$

where $c_{i \alpha}^{\dagger}$ creates an electron with spin polarization $\alpha$ at site $i$ in a honeycomb lattice, and $\langle i, j\rangle / \ll i, j \gg$ run over all the nearest/ next-nearest-neighbor hopping sites. The first term represents the nearest-neighbor hopping with the transfer energy $t$. The second term represents the chemical potential $\mu$. The third term represents the SO coupling ${ }^{29}$ with $\lambda_{\text {SO }}$, where $v_{i j}=+1(-1)$ if the next-nearestneighboring hopping is anticlockwise (clockwise) with respect to the positive $z$ axis. The fourth term represents the staggered sublattice potential term ${ }^{29}$ with $\lambda_{V}$. It may be present intrinsically ${ }^{13-15}$ or generated ${ }^{31}$ externally by applying external electric field $E_{z}$, where $\lambda_{V}=$ $\ell E_{z}$. The fifth term is the mean exchange term ${ }^{11}$ with $\lambda_{\mathrm{x}}$. The sixth term represents the staggered exchange term ${ }^{10}$ with the difference $\lambda_{\mathrm{SX}}$ between the $A$ and $B$ sites. The last term is the staggered SO term ${ }^{10}$ with $\lambda_{\text {sso. }}$.

The low-energy Dirac Hamiltonian at the $K_{\eta}$ point is ${ }^{10}$

$$
\begin{aligned}
H_{\eta}= & v_{\mathrm{F}}\left(\eta P_{x} \tau_{x}+P_{y} \tau_{y}\right)-\mu+ \\
& \lambda_{\mathrm{SO}} \eta \sigma_{z} \tau_{z}+\lambda_{V} \tau_{z}+\lambda_{\mathrm{X}} \sigma_{z}+\lambda_{\mathrm{SX}} \sigma_{z} \tau_{z},
\end{aligned}
$$

where $v_{\mathrm{F}}=\frac{\sqrt{3}}{2 \hbar}$ at is the Fermi velocity, and $P_{i} \equiv \hbar k_{i}+e A_{i}$ is the covariant momentum. We divide the potential terms into two groups, one proportional to $\tau_{z}$ and the other not. When the spin $s_{z}$ is diagonalized, they are given by $\Delta_{s_{z}}^{\eta} \tau_{z}+\mu_{s_{z}}^{\eta}$, with

$$
\Delta_{s_{z}}^{\eta}=\eta s_{z} \lambda_{\mathrm{SO}}+\lambda_{V}+s_{z} \lambda_{\mathrm{SX}}
$$

and

$$
\mu_{s_{z}}^{\eta}=-\mu+s_{z} \lambda \mathrm{X}
$$

Here, $\Delta_{s_{z}}^{\eta}$ is the Dirac mass and $\mu_{s_{z}}^{\eta}$ shifts the energy spectrum.

Electrons make cyclotron motion under perpendicular magnetic field and fill the energy levels. We evaluate the energy spectrum 


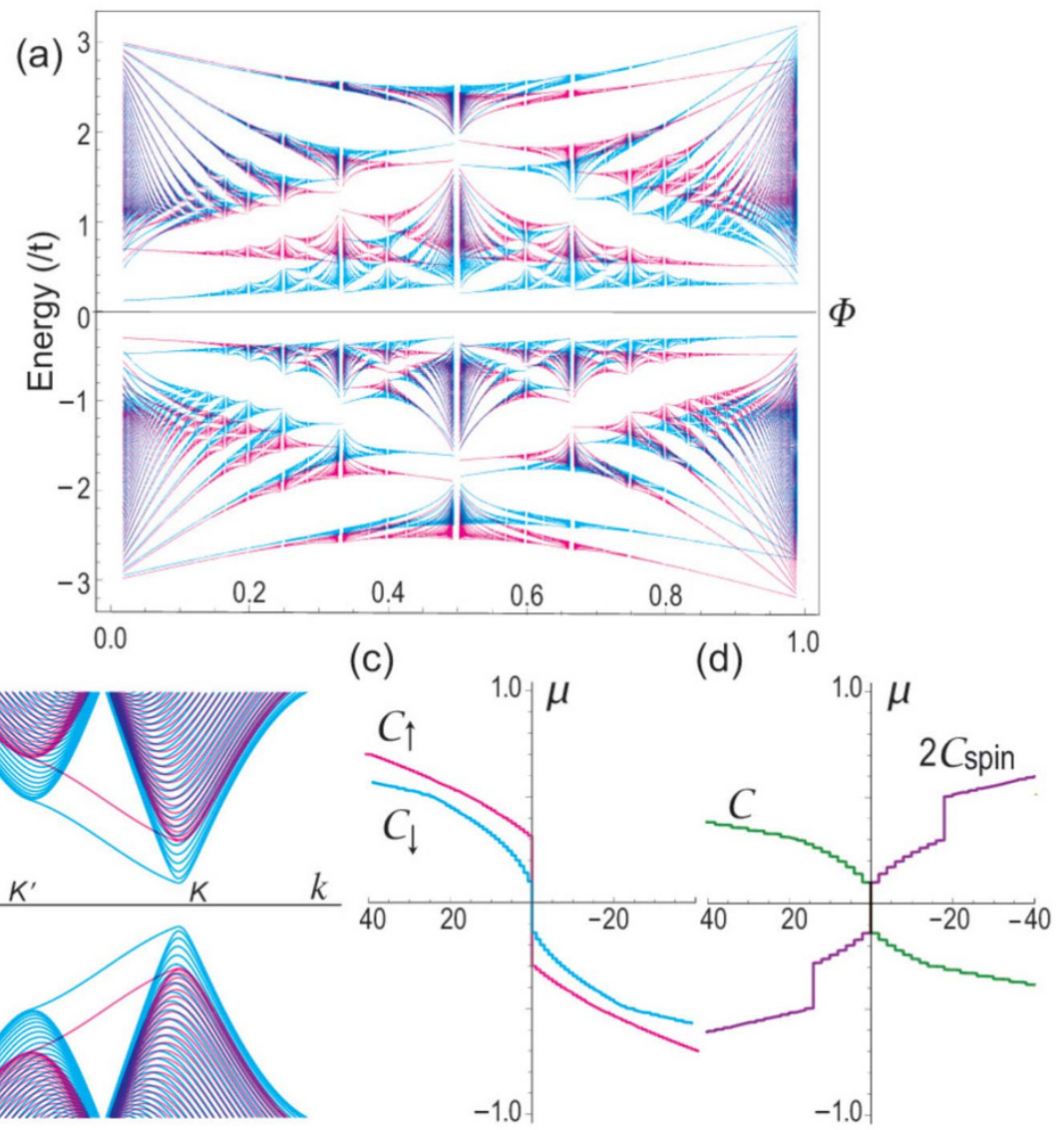

Figure $4 \mid$ TMO in electric field. We have set $\lambda_{\mathrm{SO}}=0.2 t, \lambda_{V}=0.1 t$ and $\lambda_{\mathrm{SX}}=0.4 t$ for illustration. See also the caption of Fig. 2.

numerically based on the tight-binding Hamiltonian (1) and analytically based on the low-energy Dirac theory (2). To see a global pattern of spin resolution we explore Hofstadter's butterfly diagrams $^{21-25}$ in the lattice theory. To see a detailed pattern of spin resolution in the low-magnetic field regime we explore fan diagrams in the Dirac theory. We then calculate the Chern and spin-Chern numbers based on the bulk-edge correspondence ${ }^{21}$ in the lattice theory and on the Kubo formula ${ }^{26,27}$ in the Dirac theory. They show a perfect agreement in the low-magnetic field regime. We have explicitly applied these methods to QH states and reveal high spin-Chern insulators in various honeycomb systems.

The Hall and spin-Hall conductivities are given by using the TKNN formula ${ }^{4}$ as

$$
\sigma_{x y}=\frac{e^{2}}{2 \pi \hbar} \mathcal{C}, \quad \sigma_{x y}^{\text {spin }}=\frac{e}{2 \pi \hbar} \mathcal{C}_{\text {spin }},
$$

with $^{5,6}$

$$
\mathcal{C}=\mathcal{C}_{\uparrow}+\mathcal{C}_{\downarrow}, \quad \mathcal{C}_{\text {spin }}=\frac{1}{2}\left(\mathcal{C}_{\uparrow}-\mathcal{C}_{\downarrow}\right),
$$

where $\mathcal{C}_{s_{z}}$ is the summation of the Berry curvature in the momentum space over all occupied states of electrons with $s_{z}=\uparrow \downarrow$. We summarize the results on high spin-Chern insulators in Fig. 1 for systems we have studied.

Graphene with magnetic order. The first example is given by graphene. The staggered potential is introduced by attaching boron-nitride ${ }^{32}$ or silicon-carbide on to graphene. Experimentally observed gap opening is $0.26 \mathrm{eV}$ on the $\mathrm{SiC}$ substrate ${ }^{33}$. On the other hand, the first-principle calculation shows that we can introduce ferromagnetic exchange interaction in the order of $5 \mathrm{meV}^{9,34,35}$.
We thus study the Hamiltonian (1) together with $t=2.7 \mathrm{eV}, \lambda_{V}=$ $0.26 \mathrm{eV}, \lambda_{\mathrm{X}}=5 \mathrm{meV}, \lambda_{\mathrm{SO}}=\lambda_{\mathrm{SX}}=\lambda_{\mathrm{SSO}}=0$. Due to the ferromagnetic order $\left(\lambda_{\mathrm{x}} \neq 0\right)$, the energy levels of up-spin and down-spin electrons are shifted in opposite directions, as illustrated in the band structure of a zigzag nanoribbon [Fig. 2(b)]. Thus there appear only up-spin electrons and down-spin holes near the Fermi level both for the $K$ and $K^{\prime}$ points at $\Phi=0$. The band structure also shows that the system is a trivial insulator.

The Hofstadter diagram is displayed in Fig. 2(a). Perfectly polarized up-spin electrons and down-spin holes are found all over $\Phi$. The experimentally accessible regime is given by $\Phi<10^{-3}$ or $B<16$ Tesla. We have calculated the Chern and spin-Chern numbers $\mathcal{C}$ (green) and $\mathcal{C}_{\text {spin }}$ (violet) as functions of $\mu$ at $\Phi=10^{-3}$ in Fig. 2(d). QH plateaux emerge at $v=0, \pm 1, \pm 3, \pm 5, \pm 7, \ldots$, for which the spin-Chern numbers are

$$
\mathcal{C}_{\text {spin }}=\frac{1}{2}|v|
$$

High spin-Chern insulators thus emerge. The Hall and spin-Hall conductivities are illustrated in Fig. 1(a) for $|v| \leq 10$.

Transition metal dichalcogenides with ferromagnetic order. The second example is given by monolayer transition metal dichalcogenide $\left(\mathrm{MX}_{2}\right)$. Recently there are experimental report on the ferromagnetism in $\mathrm{MX}_{2}{ }^{36,37}$. It is a trivial insulator due to a large staggered potential even though there exists a SO coupling, as is demonstrated by the band structure of nanoribbon [Fig. 3(b)]. It is described by massive Dirac fermions. Transition metal dichalcogenides have many varieties depending on the combination of transition metal and chalcogen. The examples are $\mathrm{MoS}_{2}, \mathrm{MoSe}_{2}, \mathrm{WS}_{2}, \mathrm{WS} \mathrm{S}_{2}$. Among them molybdenite $\left(\mathrm{MoS}_{2}\right)$ is a most typical material. We take the sample parameters ${ }^{13} t=$ 


\section{silicene}

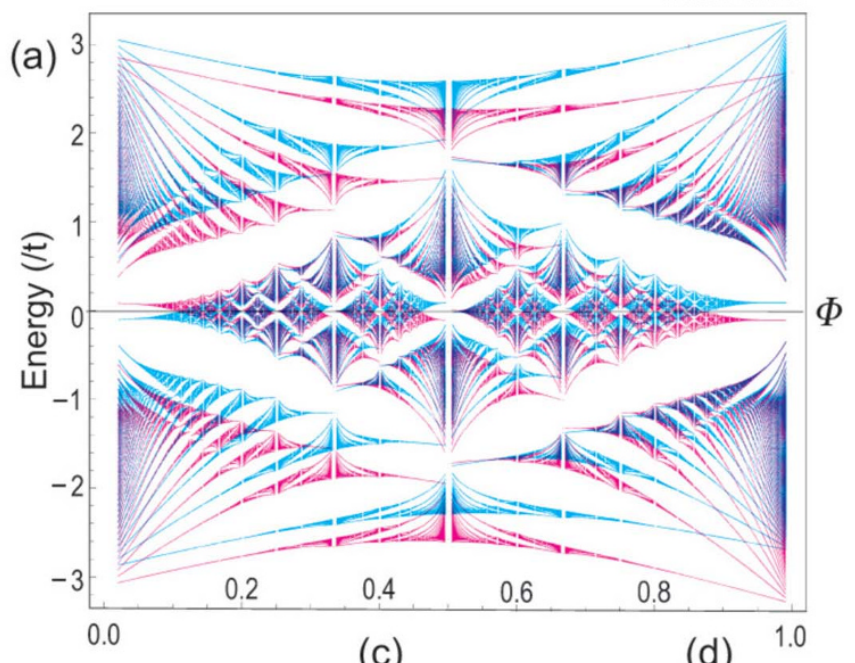

(b)

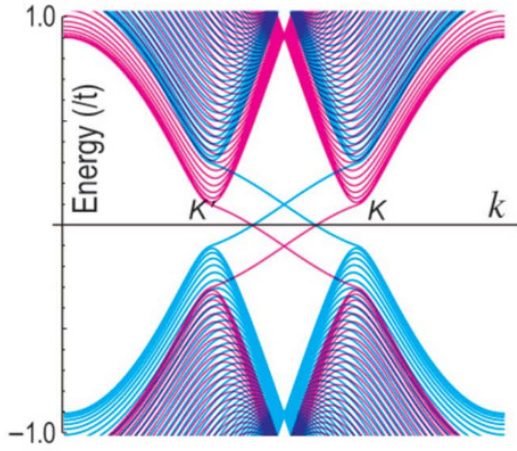

(c)

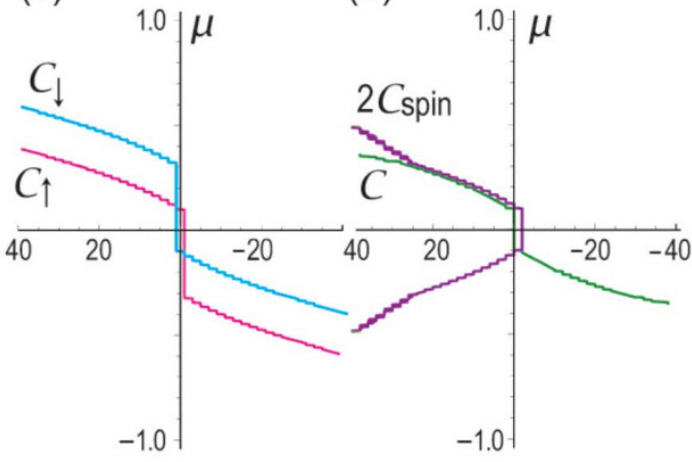

Figure $5 \mid$ Silicene with ferromagnetic order. We have set $\lambda_{\text {SO }}=0.2 t$ and $\lambda_{\mathrm{X}}=0.1 t$ for illustration. See also the caption of Fig. 2 .

$1.1 \mathrm{eV}, \lambda_{\mathrm{SO}}=\lambda_{\mathrm{sSO}}=75 \mathrm{meV}, \lambda_{V}=830 \mathrm{meV}, \lambda_{\mathrm{x}}=\lambda_{\mathrm{sx}}=0$ in the Hamiltonian (1). We note that the magnitudes of the SO term and staggered SO term are exactly identical since the intrinsic SO interaction exists only at one sublattice.

The Hofstadter diagram is displayed in Fig. 3(a). We have calculated the Chern and spin-Chern numbers $\mathcal{C}$ (green) and $\mathcal{C}_{\text {spin }}$ (violet) as functions of $\mu$ at $\Phi=10^{-3}$ in Fig. 3(d). The QH plateaux reads $v=$ $0, \pm 1, \pm 2, \pm 3, \pm 4, \ldots$, for which the spin-Chern numbers are

$$
\mathcal{C}_{\text {spin }}=\frac{1}{2}|v| \text {. }
$$

High spin-Chern insulators thus emerge. The Hall and spin-Hall conductivities are illustrated in Fig. 1(b) for $|v| \leq 10$.

Perovskite transition-metal oxides. The third example is given by TMO, where $t \approx 0.2 \mathrm{eV}, \lambda_{\mathrm{SO}}=7.3 \mathrm{meV}, \lambda_{V}=\ell E_{z}, \lambda_{\mathrm{SX}}=141 \mathrm{meV}$, $\lambda_{\mathrm{X}}=\lambda_{\mathrm{SSO}}=0$ for $\mathrm{LaCrAgO}^{17}$. A salient property is that the material contains an intrinsic staggered exchange effect $\propto \lambda_{\text {sX. }}$. It has antiferromagnetic order yielding Dirac mass. We can control the band structure by applying electric field due to the buckled structure. When the electric field is off $\left(\lambda_{V}=0\right)$, up-spin and down-spin electrons are degenerate. The degeneracy is resolved as $\lambda_{V}$ increases, and there appear only down-spin electrons and holes near the Fermi level both for the $K$ and $K^{\prime}$ points at $\Phi=0$, as found in the nanoribbon band structure [Fig. 4(b)].

The Hofstadter diagram is displayed in Fig. 4(a). We have calculated the Chern and spin-Chern numbers $\mathcal{C}$ (green) and $\mathcal{C}_{\text {spin }}$ (violet) as functions of $\mu$ at $\Phi=10^{-3}$ in Fig. 4(d). The QH plateaux reads $v=$ $0, \pm 2, \pm 4, \pm 6, \pm 8, \ldots$, for which the spin-Chern number reads

$$
\mathcal{C}_{\text {spin }}=-\frac{1}{2} v \text {. }
$$

High spin-Chern insulators thus emerge. The Hall and spin-Hall conductivities are illustrated in Fig. 1(c) for $|v| \leq 10$.

Silicene with ferromagnetic order. The final example is given by silicene, provided that we could introduce a ferromagnetic order. This could be done by a proximity coupling to a ferromagnet such as depositing $\mathrm{Fe}$ atoms to the silicene surface or depositing silicene to a ferromagnetic insulating substrate. Recently the effects of absorption of transition metal atoms are studied by the first-principle calculation, where the up and down band shifted oppositely ${ }^{38}$. We

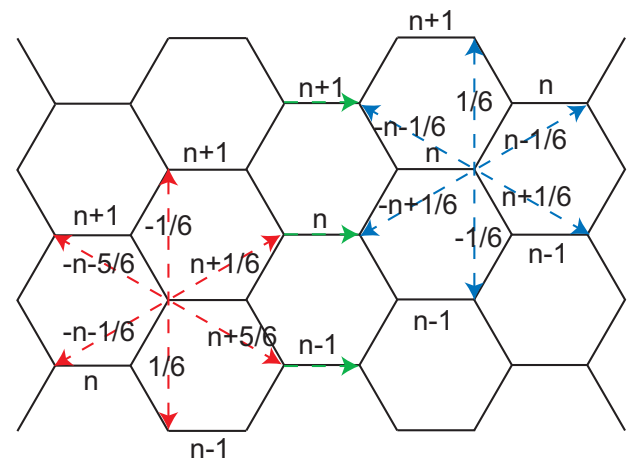

Figure 6 | Illustration of Peirls phase in honeycomb lattice. It is convenient to choose the gauge in which the Peirls phase is given by $\Phi_{i j}=$ $2 \pi \Phi l_{i j}$ between the $i$ and $j$ sites with $l_{i j}$ the number assigned to the link; $l_{i j}=n, n+\frac{1}{6}$, and so on. The horizontal links are indexed as $n=1,2, \cdots, 2 q$, with $n=1$ at the lowest corner of one magnetic unit cell. This gauge is constructed by generalizing the one used in Ref. 22 to include the link between the next-nearest neighbor sites. 
(a1) fan diagram

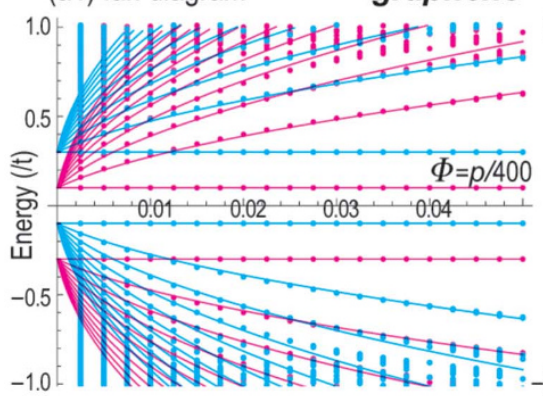

(b1)

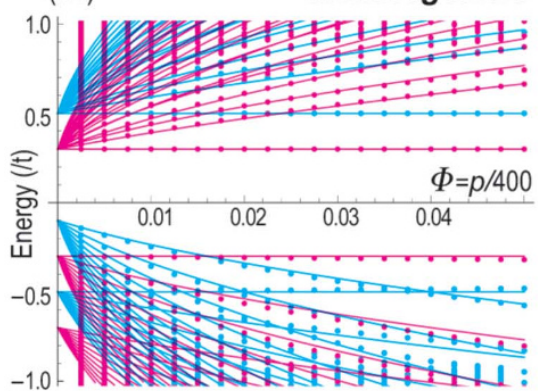

(c1)

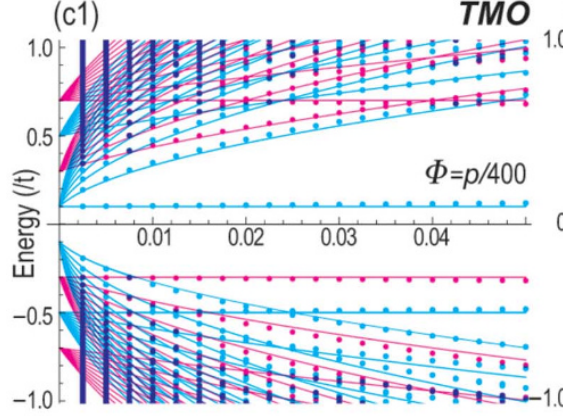

(d1)

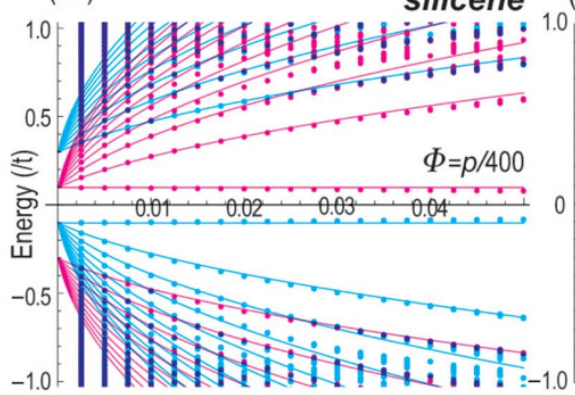

(a2) bulk-edge spectrum

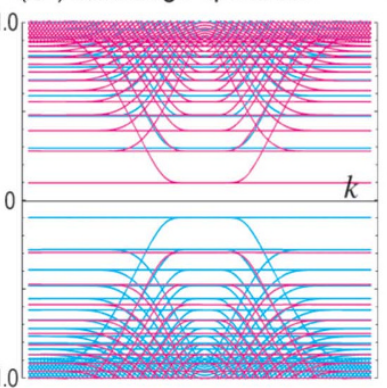

(b2)

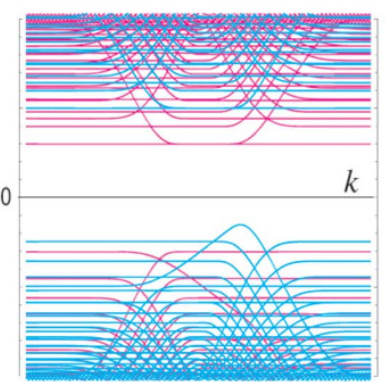

(c2)

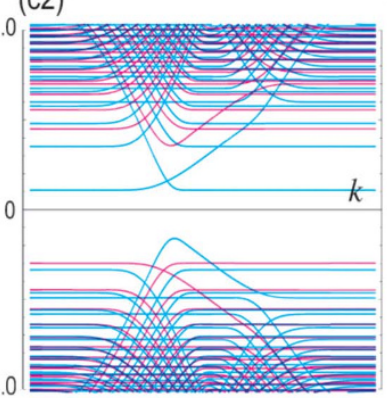

(d2)

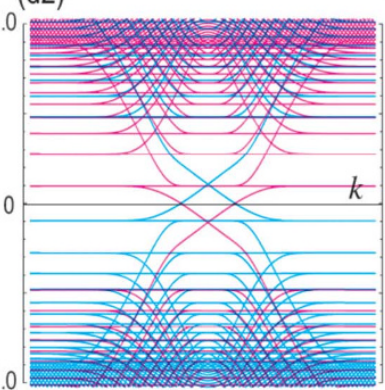

(a3) Chern numbers

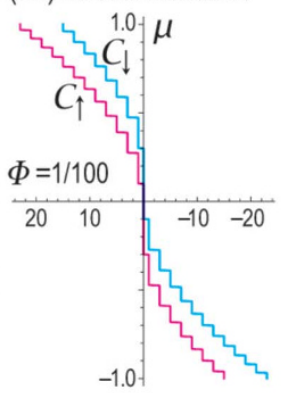

(b3)

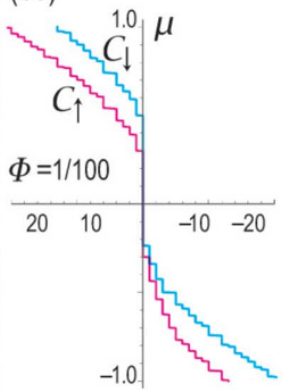

(c3)

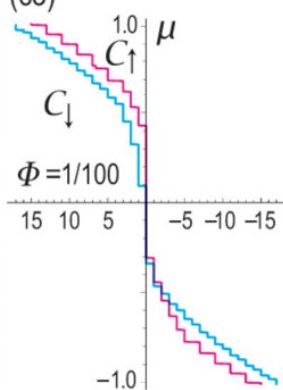

(d3)

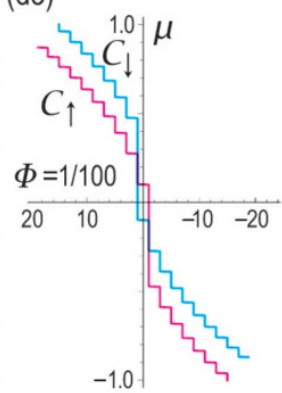

Figure $7 \mid$ Fan diagram, bulk-edge spectrum and Chern numbers. (a1 d1) Closer look of the Hofstadter's butterfly for $\Phi=p / 400$ with $p=1,2, \cdots, 20$, and the fan diagram for the Landau levels. The horizontal axis is the magnetic flux $\Phi$. (a2 $\sim \mathrm{d} 2)$ Energy spectrum with zigzag edges derived based on the lattice theory at $\Phi=1 / 100$. The horizontal axis is the momentum $k$. We can determine $\mathcal{C}_{s_{z}}$ by counting the number of edge modes. (a3 $\sim \mathrm{d} 3$ ) Chern numbers $\mathcal{C}_{s_{z}}$ calculated based on the Kubo formula (17) as a function of $\mu$ at $\Phi=1 / 100$. The horizontal axis is the topological number $\mathcal{C}_{s_{z}}$. The same results on $\mathcal{C}_{s_{z}}$ are obtained from the methods $(\mathrm{a} 2 \sim \mathrm{d} 2)$ and $(\mathrm{a} 3 \sim \mathrm{d} 3)$. See also the caption of Fig. 2.

thus have $t=1.6 \mathrm{eV}, \lambda_{\mathrm{SO}}=3.9 \mathrm{meV}, \lambda_{\mathrm{X}} \neq 0, \lambda_{V}=\lambda_{\mathrm{Sx}}=\lambda_{\mathrm{SSO}}=0$ in the Hamiltonian (1).

The nanoribbon band structure is given at $\Phi=0$ in Fig. 5(b). The spin-resolved Hofstadter diagram is displayed in Fig. 5(a). We have calculated the Chern and spin-Chern numbers $\mathcal{C}$ (green) and $\mathcal{C}_{\text {spin }}$ (violet) as functions of $\mu$ at $\Phi=10^{-3}$ in Fig. 5(d). The QH plateaux reads $v=0, \pm 2, \pm 4, \pm 6, \pm 8, \ldots$, for which the spin-Chern number reads

$$
\mathcal{C}_{\text {spin }}=\frac{1}{2}|v|-1 .
$$

High spin-Chern insulators thus emerge. The Hall and spin-Hall conductivities are illustrated in Fig. 1(d) for $|v| \leq 10$.
It is intriguing that $\mathcal{C}_{\text {spin }}=-1$ at $v=0$. This is a QSH insulator without the time-reversal symmetry. The emergence of this state is natural since silicene is a QSH insulator, to which the present system is reduced adiabatically in the limit $\Phi \rightarrow 0$. The QSH insulator under broken time-reversal symmetry has already been discussed theoretically $^{7-9,39}$ and recently found experimentally ${ }^{40}$.

\section{Discussion}

High spin-Chern insulators may arise in QH states due to strong exchange interactions generated by magnetic order. The magnetic order may be intrinsically present or introduced externally. They give 
a new type of spin-Chern insulators. Honeycomb systems are most realistic for practical materialization. We have presented four examples, graphene on the $\mathrm{SiC}$ substrate with ferromagnetic order, $\mathrm{MX}_{2}$ with ferromagnet order, TMO in electric field and silicene with ferromagnet order. Similarly these QH states may occur in other honeycomb systems such as boron-nitride and silicon carbide provided that magnetic order is introduced.

The condition for such QH states to appear is given essentially by the band structure in the absence of magnetic field. We arrange the band structure to contain either up-spin or down-spin electrons near the Fermi level by implementing appropriate magnetic order. For small magnetic field, there are many Landau levels. When there are $N$ spin-polarized energy levels, the maximum energy $\sqrt{2 \hbar v_{\mathrm{F}}^{2} e N B}$ must be smaller than the energy gap $\Delta E$ between the two Dirac cones with the opposite spins, where $\Delta E=\left|\Delta_{\uparrow}^{K}-\Delta_{\downarrow}^{K}\right|$ or $\Delta E=\left|\Delta_{\uparrow}^{K^{\prime}}-\Delta_{\downarrow}^{K^{\prime}}\right|$ with $\Delta_{s_{z}}^{\eta}$ the Dirac mass (3). The maximum value of the spin-Chern number is given by $(\Delta E)^{2} / 4 \hbar v_{\mathrm{F}}^{2} e B$, when there exists no degeneracy in the spectrum. It increases as $B$ decreases.

\section{Methods}

We have employed the following methods to make the analysis of $\mathrm{QH}$ systems in various honeycomb systems and derive their Chern and spin-Chern numbers.

Hofstadter butterfly. We compute the bulk band structure numerically by applying periodic boundary conditions to the honeycomb system. This requires that the magnetic flux $\Phi$ to be a rational number, $\Phi=p / q$ ( $p$ and $q$ are mutually prime integers). Then, the system is periodic in both spatial directions. We use the Bloch theorem to reduce the Schrödinger equation to a $2 q \times 2 q$ matrix equation for each $s_{z}$ $=\uparrow \downarrow$, where the factor 2 is due to the sublattice $(A, B)$ degrees of freedom. In so doing we choose a generalized gauge of the one used in graphene ${ }^{21}$ so as to include the link connecting the next-nearest neighbor hopping sites. It is given in such a way that the magnetic flux becomes $1 / 6$ for each isosceles triangle whose two edges are given by the neighbor hopping: See Fig. 6. The resulting band structure is the Hofstadters butterfly diagram.

We have given the Hofstadter diagram for graphene in Fig. 2(a), for dichalcogenide in Fig. 3(a), for TMO in Fig. 4(a), and for silicene in Fig. 5(a). We also present a closer look of the Hofstadter butterfly $(\Phi<0.05)$ in Fig. 7 for graphene (a1), for dichalcogenide (b1), for TMO (c1), and for silicene (d1).

Fan diagram. We introduce a pair of ladder operators,

$$
\hat{a}=\frac{\ell_{B}\left(P_{x}+i P_{y}\right)}{\sqrt{2} \hbar}, \quad \hat{a}^{\dagger}=\frac{\ell_{B}\left(P_{x}-i P_{y}\right)}{\sqrt{2} \hbar},
$$

satisfying $\left[\hat{a}, \hat{a}^{\dagger}\right]=1$, where $\ell_{B}=\sqrt{\hbar / e B}$ is the magnetic length. The Hamiltonian $H_{\eta}$ is block diagonal and given by

$$
H_{\eta}=\left(\begin{array}{cc}
H_{\uparrow}^{\eta} & 0 \\
0 & H_{\downarrow}^{\eta}
\end{array}\right),
$$

with the diagonal elements being

$$
\begin{gathered}
H_{s_{z}}^{K}=\left(\begin{array}{cc}
\Delta_{s_{z}}^{K}+\mu_{s_{z}}^{K} & \hbar \omega_{c} \hat{a}^{\dagger} \\
\hbar \omega_{c} \hat{a} & -\Delta_{s_{z}}^{K}+\mu_{s_{z}}^{K}
\end{array}\right), \\
H_{s_{z}}^{K^{\prime}}=\left(\begin{array}{cc}
\Delta_{s_{z}}^{K^{\prime}}+\mu_{s_{z}}^{K^{\prime}} & -\hbar \omega_{c} \hat{a} \\
-\hbar \omega_{c} \hat{a}^{\dagger} & -\Delta_{s_{z}}^{K^{\prime}}+\mu_{s_{z}}^{K^{\prime}}
\end{array}\right)
\end{gathered}
$$

in the basis $\left\{\psi_{A}, \psi_{B}\right\}^{t}$. Here, $\omega_{c}=\sqrt{2} v_{\mathrm{F}} / \ell_{B}$ is the cyclotron frequency.

It is straightforward to solve the eigen equation of $H_{s_{z}}^{\eta}$. The eigenvalues are

$$
E_{s_{z}}^{\eta}(N, \pm)=\mu_{s_{z}}^{\eta} \pm \sqrt{\left(\hbar \omega_{c}\right)^{2} N+\left(\Delta_{s_{z}}^{\eta}\right)^{2}}
$$

for $N=1,2, \cdots$, which depend on $\Phi$. We also have

$$
E_{s_{z}}^{\eta}(0)=\mu_{s_{z}}^{\eta}+\eta \Delta_{s_{z}}^{\eta},
$$

corresponding to $N=0$, which is independent of $\Phi$. The eigenstate describes electrons when $E_{s_{z}}^{\eta}>0$ and holes when $E_{s_{z}}^{\eta}<0$. Note that, in the energy spectrum (15a), \pm corresponds to electrons or holes provided $\mu_{s}^{\eta}$ is zero or sufficiently small.

We refer to each energy spectrum $E_{s_{z}}^{\eta}$ as a fan. There are four fans indexed by valley $K_{\eta}$ and spin $s_{z}$. Each fan consists of two parts, one for electrons and the other for holes.
These two parts are connected at one pivot when $\Delta_{s_{z}}^{\eta}=0$, and otherwise one fan has two pivots. The separation between these two pivots is given by $2 \Delta_{s_{z}}^{\eta}$, while the average distance of the two pivots from the Fermi level is given by $\mu_{s_{z}}^{\eta}$. Let us call the energy level (15b) the lowest Landau level. In this convention there exists one lowest Landau level in each fan. Thus there are four lowest Landau levels in one fan diagram.

We illustrate the fan diagram in Fig. 7 for graphene (a1), for dichalcogenide (b1), for TMO (c1), and for silicene (d1). We also present a closer look of the Hofstadter butterfly $(\Phi<5 / 100)$ on the same figure. They agree with one another very well for $\Phi$ $<1 / 100$. We can see the degeneracy of each Landau level from the fan diagram, by noting which pivots the lowest Landau levels are attached to. Thus the lowest Landau levels are nondegenerate in graphene (a1), for dichalcogenide (b1) and for TMO (c1), but 2 -fold degenerate for silicene (d1).

Buld-edge correspondence. The most convenient way to determine the topological charge in the lattice formulation is to employ the bulk-edge correspondence ${ }^{21}$. The edge-state analysis can be performed for a system with boundaries such as a cylinder. When solving the Harper equation on a cylinder, the spectrum consists of bulk bands and topological edge states. See Fig. 7(b). We typically find a few edge states within the bulk gaps, some of which cross the gap from one bulk band to another. Each edge state contributes one unit to the quantum number $\mathcal{C}_{s_{z}}$ for each $s_{z}=\uparrow \downarrow$ at the filling $v=N$. More precisely, in order to evaluate $\mathcal{C}_{s_{2}}$, we count the edge states, taking into account their location (right or left edges) and direction (up or down) of propagation ${ }^{21}$. The location of each state is derived by computing the wave function, while the direction of propagation can be obtained from the sign of its momentum derivative $d E / d k$, with $k$ the momentum parallel to the edge. We focus on one edge. Edge states with opposite directions contribute with opposite signs. The resultant formula reads

$$
\mathcal{C}_{s_{z}}=N_{\mathrm{u}}^{s_{z}}-N_{\mathrm{d}}^{s_{z}},
$$

where $N_{\mathrm{u}}^{s_{z}}$ and $N_{\mathrm{d}}^{s_{z}}$ denote the number of up- and down-moving states with spin $s_{z}$, respectively, at the right edge.

Our main task is to determine the Chern and spin-Chern numbers for each Landau level. We presents the edge-state analysis at $\Phi=1 / 100$ in Fig. 7 for graphene (a2), for dichalcogenide (b2), for TMO (c2), and for silicene (d2). According to the formula (16) we count the number of edge modes, from which we derive the topological numbers $\mathcal{C}_{\uparrow}$ (magenta) and $\mathcal{C}_{\downarrow}$ (cyan) based on (6).

Kubo formula. We use the Kubo formulation in the Dirac theory to derive the Hall conductivity for each spin $s_{z}$ in each valley $K_{\eta}$. Such a formula has been derived for graphene ${ }^{26,27}$. We may generalize it to be applicable to the Dirac system (2),

$$
\begin{aligned}
\mathcal{C}_{s_{z}}(\mu)= & \frac{1}{4} \sum_{\eta}\left[\tanh \frac{\mu_{s_{z}}^{\eta}+\Delta_{s_{z}}^{\eta}}{2 k_{\mathrm{B}} T}+\tanh \frac{\mu_{s_{z}}^{\eta}-\Delta_{s_{z}}^{\eta}}{2 k_{\mathrm{B}} T}\right]+ \\
& \frac{1}{2} \sum_{\eta, n=1}^{\infty}\left[\tanh \frac{E_{s_{z}}^{\eta}(n,+)}{2 k_{\mathrm{B}} T}+\tanh \frac{E_{s_{z}}^{\eta}(n,-)}{2 k_{\mathrm{B}} T}\right]
\end{aligned}
$$

for each spin $s_{z}$. It is straightforward to calculate $\mathcal{C}_{s_{z}}(\mu)$ as a function of the chemical potential $\mu$ with the use of formulas (3), (4) and (15a).

We show curves $\mathcal{C}_{\uparrow}(\mu)$ and $\mathcal{C}_{\downarrow}(\mu)$ at $\Phi=1 / 100$ in Fig. 7 for graphene (a3), for dichalcogenide (b3), for TMO (c3), and for silicene (d3). We can explicitly check that the Kubo formula (17) presents the correct values that are obtained based on the bulkedge correspondence. We have confirmed the validity of the Kubo formula for various systems.

1. Prange, R. E. \& Girvin, S. M. (eds.) The Quantum Hall Effect, 2nd edition, (Springer, Berlin, 1990).

2. Das Sarma, S. \& Pinczuk, A. (eds.) Perspectives in Quantum Hall Effects, (Wiley, New York, 1997).

3. Ezawa, Z. F. Quantum Hall Effects: Recent Theoretical and Experimental Developments, 3rd edition, (World Scientific, Singapore, 2013).

4. Thouless, D. J., Kohmoto, M., Nightingale, M. P. \& den Nijs, M. Quantized Hall conductance in a two-dimensional periodic potential. Phys. Rev. Lett. 49, 405 (1982).

5. Hasan, M. Z. \& Kane, C. Colloquium: Topological insulators. Rev. Mod. Phys. 82, 3045 (2010).

6. Qi, X.-L. \& Zhang, S.-C. Topological insulators and superconductors. Rev. Mod. Phys. 83, 1057 (2011)

7. Sheng, D. N., Weng, Z. Y., Sheng, L. \& Haldane, F. D. M. Quantum Spin-Hall Effect and Topologically Invariant Chern Numbers. Phys. Rev. Lett. 97, 036808 (2006).

8. Prodan, E. Robustness of the spin-Chern number. Phys. Rev. B 80, 125327 (2009)

9. Yang, Y. et al. Time-reversal-symmetry-broken quantum spin Hall effect. Phys. Rev. Lett. 107, 066602 (2011).

10. Ezawa, M. Spin-valleytronics in silicene: quantum-spin-quantum-anomalous Hall insulators and single-valley semimetals. Phys. Rev. B 87, 155415 (2013).

11. Ezawa, M. Valley-polarized metals and quantum anomalous Hall effect in silicene. Phys. Rev. Lett. 109, 055502 (2012).

12. Ezawa, M. Photo-induced topological phase transition and single Dirac-cone state in silicene. Phys. Rev. Lett. 110, 026603 (2013). 
13. Xiao, D. et al. Coupled spin and valley physics in monolayers of MoS2 and other group-VI dichalcogenides. Phys. Rev. Lett. 108, 196802 (2012).

14. Cao, T. et al. Valley-selective circular dichroism of monolayer molybdenum disulphide. Nature Communications 3, 887 (2012).

15. Li, X. et al. Coupling the valley degree of freedom to antiferromagnetic order. PNAS 110, 3738 (2013).

16. Xiao, D. et al. Interface engineering of quantum Hall effects in digital transition metal oxide heterostructures. Nature Comm. 2, 596 (2011).

17. Liang, Q.-F., Wu, L.-H. \& Hu, X. Electrically Tunable Topological State in [111] Perovskite materials with antiferromagnetic exchange field. New J. Phys. 15, 063031 (2013)

18. Guzmań-Verri, G. G. \& Voon, L. C. L. Y. Electronic structure of silicon-based nanostructures. Phys. Rev. B 76, 075131 (2007).

19. Cahangirov, S. Two- and One-Dimensional Honeycomb Structures of Silicon and Germanium. Phys. Rev. Lett. 102, 236804 (2009).

20. Rammal, R. Landau level spectrum of Bloch electrons in a honeycomb lattice. J. Physique (France) 46, 1345 (1985).

21. Hatsugai, Y. Edge states in the integer quantum Hall effect and the Riemann surface of the Bloch function. Phys. Rev. B 48, 11851 (1993).

22. Hatsugai, Y., Fukui, T. \& Aoki, H. Topological analysis of the quantum Hall effect in graphene: Dirac-Fermi transition across van Hove singularities and edge versus bulk quantum numbers. Phys. Rev. B 74, 205414 (2006)

23. Esaki, K., Sato, M., Kohmoto, M. \& Halperin, B. I. Zero modes, energy gap, and edge states of anisotropic honeycomb lattice in a magnetic field. Phys. Rev. B 80 , 125405 (2009).

24. Sato, M., Tobe, D. \& Kohmoto, M. Hall conductance, topological quantum phase transition, and the Diophantine equation on the honeycomb lattice. Phys. Rev. B 78, 235322 (2008).

25. Hasegawa, Y. \& Kohmoto, M. Quantum Hall effect and the topological number in graphene. Phys. Rev. B 74, 155415 (2006).

26. Gusynin, V. P. \& Sharapov, S. G. Unconventional integer quantum Hall effect in graphene. Phys. Rev. Lett. 95, 146801 (2005).

27. Gusynin, V. P. \& Sharapov, S. G. Transport of Dirac quasiparticles in graphene: Hall and optical conductivities. Phys. Rev. B 73, 245411 (2006).

28. Tsukazaki, A. et al. Observation of the fractional quantum Hall effect in an oxide. Nature Materials 9, 889 (2010).

29. Kane, C. L. \& Mele, E. J. Quantum spin Hall effect in graphene. Phys. Rev. Lett. 95, 226801 (2005).

30. Liu, C.-C., Jiang, H. \& Yao, Y. Low-energy effective Hamiltonian involving spinorbit coupling in silicene and two-dimensional germanium and tin. Phys. Rev. B 84, 195430 (2011).

31. Ezawa, M. Topological insulator and helical zero mode in silicene under inhomogeneous electric field. New J. Phys. 14, 033003 (2012).
32. Giovannetti, G. et al. Substrate-induced band gap in graphene on hexagonal boron nitride: Ab initio density functional calculations. Phys. Rev. B 76, 073103 (2007).

33. Zhou, S. Y. et al. Substrate-induced bandgap opening in epitaxial graphene. Nature Materials 6, 770 (2007).

34. Qiao, Z. et al. Quantum anomalous Hall effect in graphene from Rashba and exchange effects. Phys. Rev. B 82, 161414 R (2010).

35. Tse, W. K. et al. Quantum anomalous Hall effect in single-layer and bilayer graphene. Phys. Rev. B 83, 155447 (2011).

36. Tongay, S. Magnetic properties of MoS2: Existence of ferromagnetism. Appl. Phys. Lett. 101, 123105 (2012).

37. Gao, D. Ferromagnetism in freestanding $\mathrm{MoS}_{2}$ nanosheets. Nanoscale Research Letters 8, 129 (2013).

38. Sahin, H. \& Peeters, F. M. Adsorption of alkali, alkaline-earth, and 3d transition metal atoms on silicene. Phys. Rev. B 87, 085423 (2013).

39. Beugeling, W., Goldman, N. \& Smith, C. M. Topological phases in a twodimensional lattice: Magnetic field versus spin-orbit coupling. Phys. Rev. B 86, 075118 (2012).

40. Du, L., Knez, I., Sullivan, G. \& Du, R. R. Observation of Quantum Spin Hall States in InAs/GaSb Bilayers under Broken Time-Reversal Symmetry. cond-mat/ arXiv:1306.1925.

\section{Acknowledgments}

I am very much grateful to $\mathrm{N}$. Nagaosa and $\mathrm{H}$. Aoki for many fruitful discussions on the subject. This work was supported in part by Grants-in-Aid for Scientific Research from the Ministry of Education, Science, Sports and Culture No. 22740196.

\section{Author contributions}

M.E. performed all calculations and made all contribution to the preparation of this manuscript.

\section{Additional information}

Competing financial interests: The authors declare no competing financial interests. How to cite this article: Ezawa, M. High Spin-Chern Insulators with Magnetic Order. Sci. Rep. 3, 3435; DOI:10.1038/srep03435 (2013).

\footnotetext{
(c) (i) $(-)$ This work is licensed under a Creative Commons Attribution-

NonCommercial-NoDerivs 3.0 Unported license. To view a copy of this license, visit http://creativecommons.org/licenses/by-nc-nd/3.0
} 